\title{
Effectiveness of educational intervention on knowledge regarding cervical cancer and its prevention among married women in Chandragiri Municipality, Kathmandu
}

\author{
Shrestha $B^{1}$ iD $ه$, Paudel $N^{2}$ iD
}

${ }^{1}$ Bipsana Shrestha, B.Sc. Nursing Graduate, Kathmandu Medical College Teaching Hospital, Kathmandu, Nepal; ${ }^{2}$ Narayani Paudel, Associate Professor, Department of Nursing, Kathmandu Medical College Teaching Hospital, Kathmandu, Nepal.

\begin{abstract}
Background: Cervical cancer is the leading cancer and the leading cause of cancer deaths in women in low and middleincome countries. There is lack of knowledge regarding cervical cancer and its prevention among Nepalese women which leads to inadequate screening. Although cervical cancer is acknowledged as a preventable disease, it is still a major health burden for women in many developing countries because an adequate scale of screening programs is lacking.

Objective: To evaluate the effectiveness of educational intervention on knowledge regarding prevention of cervical cancer among married women in Chandragiri Municipality.

Methodology: Pre-experimental research design (One-group pretest-posttest design) was used. Through nonprobability purposive sampling, 65 married women of Chandragiri municipality, ward no. 04 were included in the study. The final sample size was 62 as three samples were lost during post-test. Structured questionnaire was used for data collection via an interview method. The educational intervention was administered after pretest. Posttest was done with the same instrument two weeks after intervention. Data analysis was done using SPSS version 20. Descriptive statistics and inferential statistics (paired t-test) were used, hypothesis was tested at $5 \%$ level of significance.

Results: Out of 62 respondents, $54.8 \%$ of the respondents had adequate knowledge in the pre-intervention phase and $62.9 \%$ had adequate knowledge in the post-intervention phase regarding cervical cancer and its prevention. There was a significant increase in mean score of overall knowledge from 15.82 to 25.75 after educational intervention $(p<0.001)$. Conclusion: Mean knowledge score of the respondents increased significantly after educational intervention indicating that the educational intervention was effective.
\end{abstract}

Key words: Cervical cancer; Educational intervention; Married women; Prevention

Access this article online

Website: www.jkmc.com.np

DOI: https://doi.org/10.3126/jkmc.v9i2.35530

\section{HOW TO CITE}

Shrestha B, Paudel N. Effectiveness of educational intervention on knowledge regarding cervical cancer and its prevention among married women in Chandragiri Municipality, Kathmandu. J Kathmandu Med Coll. 2020;9(2):96-101.

Address for correspondence

Bipsana Shrestha

Nursing Officer

Bhaktapur Hospital, Bhaktapur, Nepal

E-mail: bipsanashrestha@gmail.com

Copyright @ 2020 Journal of Kathmandu Medical College (JKMC)

ISSN: 2019-1785 (Print), 2091-1793 (Online)

(i) (S) This work is licensed under a Creative Commons Attribution-Non Commercial 4.0 International License.

\section{INTRODUCTION}

Cervical cancer occurs when the cells of the cervix grow abnormally and invade other tissues and organs of the body. When it is invasive, this cancer affects the deeper tissues of the cervix and may have spread to other parts of the body (metastasis), most notably the lungs, liver, bladder, vagina and rectum ${ }^{1}$. The risk factors related to the development of cervical cancer includes infection with high-risk human papilloma virus (HPV type 16 and 18), initiation of sexual activity in early age, multiparity, multiple sexual partners, co-infection with human immunodeficiency virus (HIV) type-2, immunosuppressant and certain dietary deficiencies ${ }^{2}$.

Cervical cancer is the fourth most frequent cancer in women. In 2018 it represented $6.6 \%$ of all female cancers with an estimated 5,70,000 new cases. In lowand middle-income countries, approximately $90 \%$ of 
deaths occurred from cervical cancer A comprehensive approach that includes prevention, early diagnosis, screening and treatment programs can be effective in reducing high mortality rate from cervical cancer globally ${ }^{3}$.

Cervical cancer is the most common cancer present in Nepal. According to the Hospital Based Cancer Registry, 6249 (20.9\%) new cervical cancer has been diagnosed from 2003-2012 which is the highest among the total female new cancer cases $^{4}$. In Nepal, every year estimated 2,942 women are diagnosed with cervical cancer and 1,928 die from this disease ${ }^{5}$. Cervical cancer deaths in Nepal in 2017 was 1,624 or $0.99 \%$ of total deaths according to the latest data published by WHO which ranked Nepal $55^{\text {th }}$ in the world ${ }^{6}$.

The national guidelines on cervical cancer screening and prevention (2010) stated screening at least 50\% of women aged 30-60 years and reducing the cervical cancer mortality by $10 \%$ with recommended screening among this group every five years ${ }^{7}$.

A qualitative study done among women in Nepal showed poor knowledge about cervical cancer screening. The obstacles to attend the screening centers were found to be sociocultural barriers, service provider's behavior, geographical challenges and limited finances. Whereas, awareness programs and support from family and women's groups were taken as facilitating factors which convinced the women to attend those screening clinics ${ }^{8}$. Prior research shows a positive association between a woman's knowledge on cervical cancer screening and her likelihood of obtaining a pap smear'. The lowest prevalence of cervical cancer screening is among the illiterate and those living in rural areas. Therefore, educational campaigns and rural outreach are needed to boost screening rates ${ }^{10}$.

The objective of this study is to find out the effectiveness of educational intervention on knowledge regarding prevention of cervical cancer among married women by comparing the knowledge before and after the educational intervention.

There will be no difference in the knowledge on prevention of cervical cancer of women before and after educational intervention (Null hypothesis).

There will be a significant increase in the knowledge of women on prevention of cervical cancer after educational intervention (Research hypothesis).

\section{METHODOLOGY}

Pre-experimental (one group pretest posttest) design was used to fulfill the aim of this study. In this design, a single group of participants is observed at two time points, one before the intervention and one after the intervention. Changes in the outcome of interest are presumed to be the result of the intervention. No control or comparison group is employed.

Study was carried out in the Chandragiri municipality, ward no. 04. It took 9 weeks for the completion of the study. Study was conducted after the approval of the Institutional Review Committee of Kathmandu Medical College (Ref.:310520198). The permission was obtained from the ward office of Chandragiri municipality ward no. 03 for pretesting in $10 \%$ of sample size. Similarly, permission was also obtained from the ward office of Chandragiri municipality ward no. 04 to carry out the study. Informed consent was taken from each participant. Purpose and objectives of the study was explained to the participants. Their participation was voluntary and they had right to withdraw from the study at any time. Human dignity, privacy, confidentiality and anonymity was maintained throughout the study. The information provided by the respondents was used for the purpose of study only.

Non-probability purposive sampling technique was used to fulfill the purpose of this study. Sample size was 60.5 calculated from power analysis. The sample size 65 was taken for the study achieved after adding 5\% of the calculated sample size. (considering the fact that pretested samples might be lost during the posttest). 3 samples were lost during the post test. Thus, final sample size for the analysis was 62 .

Data collection procedure in this study involved three steps. The first step was the pre-intervention phase in which cross-sectional baseline information of the individual participant was collected through face to face interview using a self-structured questionnaire. The study instrument comprised socio-demographic information, knowledge regarding cervical cancer, signs and symptoms and its prevention. Each question had a score for the correct response and no score for the incorrect response. There were some multiple-choice questions as well.

In the second step, respondents were divided into 3 groups of about 20 participants in each group. Around 30 minutes long the educational intervention was provided by the researcher herself to the participants 
that included health education on cervical cancer and Pap smear test. The health teaching was provided in the nearby public places of Chandragiri municipality by gathering the participants in groups.

In step three, a post-intervention test was carried out 2 weeks after the intervention. The same instrument that was used for data collection in the pre-intervention phase was administered. Due to the time limitation, the posttest after intervention could not be done later than 2 weeks which may have affected knowledge retention among participants.

After the completion of data entry, the file was analyzed using SPSS (Statistical Package for the Social Sciences) version 20. Descriptive (frequency, percentage, mean, standard deviation) and inferential statistics (paired t-test) was used. Every question had a score so they were rated individually then the total score of all the respondents were calculated for pre as well as post intervention. After that, the mean score was calculated and then the score equal to or above the mean was considered adequate while those below the mean were considered inadequate.

Women who were diagnosed with cervical cancer were excluded from the study.

\section{RESULTS}

About 39\% of the respondents were of age group 30-39 years with the mean age of 41.15 years. According to the ethnicity, the majority of the respondents $(79 \%)$ were Newar (Table 1).

Table 2 shows that only $12.9 \%$ of the respondents knew the actual meaning of cervical cancer at pre-intervention phase while it increased to $45.2 \%$ at post-intervention phase. It also shows that $82.3 \%$ of the respondents knew that early marriage and having too many children are the risk factors for cervical cancer in the pre-intervention phase while it increased to $100 \%$ post-intervention.

More than half the respondents knew that irregular menstruation is one of the symptoms of cervical cancer and by the end of study all of them knew that. And most of the respondents (82.3\%) knew that abnormal foul vaginal discharge is one of the symptoms of cervical cancer even before intervention and it raised to $95.2 \%$ after intervention. However, only $17.7 \%$ of the respondents knew post coital bleeding as the symptom of cervical cancer which rose to $58.1 \%$ after intervention. Similarly, nearly half of the respondents (46.8\%) knew that vaginal bleeding after menopause is also one of the symptoms of cervical cancer and it raised to $77.4 \%$ after intervention.

Table 3 shows that the majority (91.9\%) of the respondents knew that maintaining perineal hygiene is one of the preventive measures of cervical cancer at pre-intervention phase and it increased to $100 \%$ at postintervention phase. Also, the knowledge of respondents about avoiding multiple sexual partners and using condom during sexual intercourse increased which not only prevents cervical cancer but other sexually transmitted infections as well.

Only few of the respondents knew about the HPV vaccine (8.1\%) and none of them knew about the appropriate age to take the vaccine. But after the intervention, $90.3 \%$ of the respondents knew about the vaccine while $35.5 \%$ knew about the appropriate age to take the vaccine.

Most of the people (75.8\%) knew that pap smear is most effective to prevent cervical cancer although only one of the respondents knew about the appropriate time to start pap smear. However, after the intervention, all of them knew that pap smear is most effective to prevent cervical cancer while $71 \%$ of them also knew about the appropriate time to start pap smear.

The findings of the study also revealed that $47 \%$ of the respondents had done Pap smear test at least once in their life while $53.2 \%$ of the respondents had never done Pap smear test.

Table 4 shows that there was a significant increase in mean score of the respondents from 15.82 to 25.75 after educational intervention $(p<0.001)$. Here, adequate knowledge equals to or greater that mean knowledge and inadequate knowledge means below the mean knowledge score. 
Table 1: Distribution of respondents according to the demographic variables $(n=62)$

\begin{tabular}{|c|c|c|}
\hline Variables & Frequency & Percent (\%) \\
\hline \multicolumn{3}{|l|}{ Age (Years) } \\
\hline $20-29$ & 4 & 6.5 \\
\hline $30-39$ & 24 & 38.7 \\
\hline $40-49$ & 21 & 33.9 \\
\hline $50-59$ & 13 & 21 \\
\hline \multicolumn{3}{|l|}{ Mean $\pm S D=41.15 \pm 8.11$} \\
\hline \multicolumn{3}{|l|}{ Ethnicity } \\
\hline Brahmin and Chhetri & 7 & 11.3 \\
\hline Newar & 49 & 79 \\
\hline Others & 6 & 9.7 \\
\hline \multicolumn{3}{|l|}{ Religion } \\
\hline Hinduism & 59 & 95.2 \\
\hline Others & 3 & 4.8 \\
\hline \multicolumn{3}{|l|}{ Occupation } \\
\hline Homemaker & 41 & 66.1 \\
\hline Service and others & 13 & 21 \\
\hline Business & 8 & 12.9 \\
\hline \multicolumn{3}{|l|}{ Educational status } \\
\hline Illiterate and non-formal & 21 & 33.9 \\
\hline Up to Secondary & 34 & 54.9 \\
\hline Higher secondary and above & 7 & 11.3 \\
\hline
\end{tabular}

Table 2: Correct response regarding meaning, risk factors and signs and symptoms of cervical cancer $(n=62)$

\begin{tabular}{lcc}
\hline Variables & Pre-intervention frequency (\%) & Post-intervention frequency (\%) \\
$\begin{array}{l}\text { Correct meaning of cervical cancer } \\
\text { Most common cancer in female in Nepal }\end{array}$ & $8(12.9)$ & $28(45.2)$ \\
Yes & $48(77.4)$ & $61(98.4)$ \\
Risk factors & $51(82.3)$ & $62(100)$ \\
$\quad$ Early marriage & $51(82.3)$ & $62(100)$ \\
Too many children & $34(54.8)$ & $60(96.8)$ \\
Prolonged use of OCPs & & $62(100)$ \\
Signs and symptoms & $42(67.7)$ & $53(85.5)$ \\
Irregular menstruation & $27(43.5)$ & $59(95.2)$ \\
Excessive blood loss during menstruation & $51(82.3)$ & $60(96.8)$ \\
Abnormal foul vaginal discharge & $33(53.2)$ & $61(98.4)$ \\
Weight loss & $44(71)$ & $36(58.1)$ \\
Painful micturition & $11(17.7)$ & $48(77.4)$ \\
Post coital bleeding & $29(46.8)$ & \\
Vaginal bleeding after menopause & & \\
\hline
\end{tabular}

Table 3: Correct response regarding preventive measures of cervical cancer $(n=62)$

\begin{tabular}{|lcc|}
\hline \multicolumn{1}{|c}{ Variables } & $\begin{array}{c}\text { Pre-intervention frequency } \\
\text { (\%) }\end{array}$ & $\begin{array}{c}\text { Post-intervention } \\
\text { frequency (\%) }\end{array}$ \\
\hline Avoiding multiple sexual partners & $38(61.3)$ & $62(100)$ \\
\hline Use of condom during sexual intercourse & $35(56.5)$ & $60(96.8)$ \\
\hline Preventing sexually transmitted infections & $32(51.6)$ & $49(79)$ \\
\hline Avoiding early child birth & $46(74.2)$ & $62(100)$ \\
\hline Maintaining perineal hygiene & $57(91.9)$ & $62(100)$ \\
\hline Avoiding smoking, alcohol and tobacco & $46(74.2)$ & $56(90.3)$
\end{tabular}


Table 3 cont ...

Eating fresh fruits and vegetables

51 (82.3)

59 (95.2)

5 (8.1)

56 (90.3)

Appropriate time to take HPV vaccine

$0(0)$

22 (35.5)

People who knew about Pap smear test

52 (83.9)

62 (100)

Pap smear is most effective to prevent cervical cancer.

47 (75.8)

$62(100)$

Purpose of Pap smear test

40 (64.5)

$59(95.2)$

Pap smear is essential for sexually active women

34 (54.8)

62 (100)

Appropriate time to start Pap smear

1 (1.6)

44 (71)

Time interval in which Pap smear test should be repeated.

2 (3.2)

$54(87.1)$

Appropriate time for Pap smear is 7 days after menstruation

26 (41.9)

$53(85.5)$

Pap test is not required after total hysterectomy.

$40(64.5)$

$61(98.4)$

Table 4: Comparison of pre-intervention and post-intervention mean knowledge score regarding cervical cancer and its prevention $(n=62)$

\begin{tabular}{cccc} 
Knowledge & Pre-intervention & Post-intervention & p-value \\
Mean \pm SD & $15.82 \pm 3.9$ & $25.75 \pm 2.69$ & $<0.001^{*}$ \\
Adequate & $34(54.8 \%)$ & $39(62.9 \%)$ & $23(37.1 \%)$ \\
Inadequate & $28(45.2 \%)$ & 23 & \\
\hline
\end{tabular}

${ }^{*}$ t-test significant at $<0.001$

\section{DISCUSSION}

In this study, $38.7 \%$ of the respondents were of age group 30-39 years with the mean age of 41.15 years. Majority (95.2\%) of the respondents followed Hinduism as a religion and $66.1 \%$ of the respondents were homemakers. This was supported by a similar study done on women attending gynae OPD of a Teaching hospital, Bharatpur, Chitwan where 58.3\% were of age group 3039 years with the mean age of 38.83 . Majority (90.6\%) of the respondents followed Hindu religion and $59.4 \%$ of the respondents were homemaker ${ }^{4}$.

The findings of this study showed that $54.8 \%$ of the respondents had adequate knowledge in the preintervention phase while $45.2 \%$ had inadequate knowledge regarding prevention of cervical cancer. It was inconsistent with the results of a study conducted in Teaching Hospital, Bharatpur, Chitwan which showed that $34.4 \%$ of respondents had adequate and $65.6 \%$ had inadequate knowledge 4 . The difference in knowledge score could be due to the fact that a local organization in the Chandragiri municipality has been conducting programmes related to cervical cancer time and again.

The findings of this study showed $62.9 \%$ of the respondents had adequate knowledge in the postintervention phase while $37.1 \%$ had inadequate knowledge regarding cervical cancer and its prevention which shows that there was improvement in the knowledge of the respondents after intervention.
Similar findings were observed in a study conducted in an outpatient clinic affiliated at obstetric department at Benha University Hospital in which it was mentioned that $64.6 \%$ had good, $26.2 \%$ had average and $9.2 \%$ had poor knowledge regarding cervical cancer during post intervention phase ${ }^{11}$.

A study conducted to improve HPV and cervical cancer knowledge among African American college students revealed that $96 \%$ of students reported knowledge of the HPV vaccine ${ }^{12}$. Whereas in our study only $8.1 \%$ of respondents knew about the HPV vaccine in the preintervention phase although it increased to 90.3 after educational intervention. Nepalese people are unaware about the HPV vaccine may be because most of them are not vaccinated against HPV since it is one of the expensive vaccines and it is not under the regular vaccination list of the Nepalese government.

In this study, the mean knowledge score regarding cervical cancer and its prevention of the respondents increased from 15.82 to 25.75 after educational intervention which is in line with the result of the previously mentioned study conducted at Benha University Hospital in which mean score increased from 11.33 to 21.2 after educational intervention ${ }^{11}$. Similarly, the study conducted to improve HPV and cervical cancer knowledge among African American college students revealed that correct response rates significantly increased with the intervention ${ }^{12}$. The educational 
intervention was found to be effective in increasing the knowledge of the respondents in all three of these studies as the difference between pre-intervention and postintervention knowledge was found to be statistically significant. In contrast, a study conducted in Valparaiso, Indiana revealed that the mean pre-intervention score of knowledge was 5.10 that increased up to 5.35 after intervention. It reflects an improvement in knowledge based on the intervention, but the difference was not statistically significant. The high pre- intervention scores may be reflective of the high level of education among the participants, as $98.6 \%(n=30)$ had at least a high school education, and $74.2 \%(n=23)$ had at least 2 years of college education ${ }^{13}$.

The findings of this study might be beneficial to women of reproductive age to involve in screening program of cervical cancer thereby decreasing the mortality and morbidity. This may also be useful to the health institutions as well as Non-Governmental Organizations (NGOs) and International Non-Governmental Organizations (INGOs) to provide educational programs on prevention of cervical cancer among females of

\section{REFERENCES}

1. Cervical Cancer. WebMD. [Internet]. 2019 [cited 2019 Apr 22]. [DOI]

2. Gana GJ, Oche MO, Ango JT, Kaoje AU, Awosan KJ, Raji IA. Educational intervention on knowledge of cervical cancer and uptake of Pap smear test among market women in Niger State, Nigeria. J Public Health Africa [Internet]. 2017 Dec 31 [cited 2019 Apr 21];8(2):575. [PubMed]

3. WHO.Cervical cancer [Internet]. World Health Organization; 2018 [cited 2019 Apr 21]. [DOI]

4. Shrestha S, Dhakal P. Knowledge, Attitude and Practice Regarding Cervical Cancer Screening Among Women Attending a Teaching Hospital, Bharatpur, Chitwan. J Fam Reprod Health [Internet]. 2017 Mar [cited 2019 Apr 21];11(1):18-23. [PubMed]

5. HPV Information Centre. Complementary data on cervical cancer prevention. Catalan Institute of Oncology, International Agency for Research on Cancer [Internet]. [cited 2019 Apr 21].t [DOI]

6. World Health Rankings. Cervical Cancer in Nepal [Internet]. 2018 [cited 2019 Apr 21]. [DOI]

7. Ministry of Health Government of Nepal. Annual Report Department of Health Services 2072/2073 (2015/2016) [Internet]. Vol. 73. 2017. [Full text]

8. Darj E, Chalise P, Shakya S. Barriers and facilitators to cervical cancer screening in Nepal: A qualitative different communities. Policy makers can use the results of the study to improvise policies to maximize screening for cervical cancer which in time will help to curb down cervical cancer mortality and morbidity.

Since the purposive sampling technique was used, findings of the study may not be generalized. Due to the time limitation, the posttest after intervention could not be done later than 2 weeks which may have affected knowledge retention among participants.

\section{CONCLUSION}

The mean knowledge score of the respondents increased after educational intervention which indicates that the educational intervention was effective. Therefore, educational packages regarding cervical cancer and its prevention should be conducted time and again in different communities. Awareness programs related to cervical cancer screening should be encouraged at local level through the medium of health personnel, family members and friends, television, radio and newspapers. Health camps relating cervical cancer screening can also be conducted at the community level.

study. Sex Reprod Healthc [Internet]. 2019 Jun 1 [cited 2019 Apr 23];20:20-6. [DOI]

9. Perkins RB, Langrish S, Stern LJ, Simon CJ. A community-based education program about cervical cancer improves knowledge and screening behavior in Honduran women. Rev Panam Salud Pública [Internet]. 2007 Sep [cited 2019 Apr 23];22(3):187-93. [FullText]

10. Ranjit A, Gupta S, Shrestha R, Kushner AL, Nwomeh $B C$, Groen RS. Awareness and prevalence of cervical cancer screening among women in Nepal. Int J Gynecol Obstet [Internet]. 2016 July 1 [cited 2019 Apr 21];134(1):37-40. [DOI]

11. Said $S A E$, Hassan $H E$, Sarhan AEM. Effect of an Educational Intervention on Women's Knowledge and Attitude Regarding Cervical Cancer. Am J Nurs Res [Internet]. 2018 Mar10 [cited 2019 Apr 21];6(2):59-66. [DOI]

12. Staples J, Wong M. An Educational Intervention to Improve HPV and Cervical Cancer Knowledge Among African American College Students. Obstet Gynecol [Internet]. 2017 Oct [cited 2019 Apr 23];130:45S. [DOI]

13. Trapp AM. ValpoScholar The Effect of an Educational Intervention on Knowledge and Intent to Participate in Cervical Cancer Screening [Internet]. 2015 [cited 2019 Apr 23]. [DOI] 